\title{
Fatigue detection through physiological assessment during real-life occupational situations: Preliminary results
}

\author{
D. Bustos ${ }^{1}$, J.C. Guedes 2 , P. Pratas ${ }^{3}$, M.P. Vaz ${ }^{4}$, J.C. Torres Costa ${ }^{5}$, R.J. Fernandes ${ }^{6}$ and J. Santos Baptista
}

\begin{abstract}
Associated Laboratory for Energy, Transports and Aeronautics - LAETA (PROA), Faculty of Engineering, University of Porto, PT (Idbs@ fe.up.pt) ORCID 0000-0002-4942-7625, 2Associated Laboratory for Energy, Transports and Aeronautics - LAETA (PROA), Faculty of Engineering University of Porto, PT (jccg@fe.up.pt) ORCID 0000-0003-2367-2187, ${ }^{3}$ Faculty of Engineering, University of Porto, PT (up202003606@fe.up.pt) ORCID 0000-0003-3380-5027, 4Associated Laboratory for Energy, Transports and Aeronautics - LAETA (PROA), Faculty of Engineering, University of Porto, PT; Porto Biomechanics Laboratory, University of Porto, PT (gmavaz@fe.up.pt) ORCID 0000-0002$6347-9608,{ }^{5}$ Associated Laboratory for Energy, Transports and Aeronautics - LAETA (PROA), Faculty of Medicine, University of Porto, PT (zecatoco@sapo.pt) ORCID 0000-0003-3947-8688, ${ }^{6}$ Center of Research, Education, Innovation and Intervention in Sport, Faculty of Sport, University of Porto, PT; Porto Biomechanics Laboratory, University of Porto, PT (ricfer@fade.up.pt) ORCID 0000-0002-5811-0443, ${ }^{7}$ Associated Laboratory for Energy, Transports and Aeronautics - LAETA (PROA), Faculty of Engineering, University of Porto, PT (jsbap@fe.up.pt) ORCID 0000-0002-8524-5503

https://doi.org/10.24840/978-972-752-279-8 0049-0055
\end{abstract}

\begin{abstract}
Background: Fatigue is a significant health and safety-related problem among workers. In general, it decreases performance and physical strength, causing incidents and accidents in operational situations. During military activities, soldiers often encounter severe conditions, which combined lead to fatigue manifestations affecting their health and performance. Continuous monitoring of their overall health status would prevent its adverse effects. Objective: This work aimed to present the preliminary results of a retrospective assessment of military training physiological recordings using an alert-based fatigue detection algorithm to validate its accurate functioning. Methods: Three case studies from soldiers participating in military training tests were recruited for evaluation. The referred algorithm was developed to manage fatigue through the combined assessment of physiological variables and determine different fatigue levels warnings to advise timely interventions and prevent potential health impact. Each examined case included the continuous recording of heart rate, breathing rate and core temperature. The algorithm translated physiological sensory data into minute alarms according to fatigue levels determined through the conjunction of normative and related research criteria. Results and Discussion: Outcomes revealed that the algorithm could evidence the different stages of training and the resulting physical demands on soldiers using their physiological response throughout the exercises. Retrieved fatigue alarms showed the high physiological cost of military practices and helped to overview the impact of each training period. Finally, results also demonstrated the importance of individual and contextualised assessment for accurately characterise the subject's fatigue status. Conclusions: It is concluded that the developed decision model can improve the management of real-time fatigue, allowing early detection of potential indicators of further physical impairments. Furthermore, it can lead to the enhancement of work-rest cycles, not only for tactical personnel but also for any safety-sensitive occupation. For future work, its validity will be tested through more participants, and other variables will be added to improve its accuracy.
\end{abstract}

Keywords: Performance, Physical exertion, Physiological monitoring, Sensors.

\section{INTRODUCTION}

Fatigue is a major health and safety-related challenge among workers (Anwer et al., 2021). In general, it can be understood as a reduction in physical and mental capacity to perform activities at the desired level due to exhaustion of mental or physical strength (Hallowell, 2010; Ream \& Richardson, 1996). Fatigue degrades performance and well-being, leading to error, incident, and accident in operational settings (Belenky et al., 2014). Because of the potential influence of fatigue on health, safety, and productivity, any organisation in which individuals work extended hours, or hours in which people typically sleep, can experience fatigue in the workplace. This is especially important for safety-sensitive operations such as the transportation, military missions, health care, and energy industries (Lerman et al., 2012).

Fatigue is widely prevalent among military personnel. Besides typical operational stressors, soldiers, potentially more than any other working group, must deal with stressful situations that can lead to a state of fatigue, non-functional overreaching, and eventually overtraining conditions (Friedl, 2012; Parnell et al., 2018). As a result, early detection and real-time monitoring of fatigue play vital roles in the military (Friedl, 2012; Stacey et al., 2018). In general, literature has evidenced that traditional methods to assess fatigue, such as subjective 
measurements and self-reported fatigue (Fang et al., 2015; Lee et al., 1991; Rachmawati et al., 2020; Zhang et al., 2015), are biased by situational and individual perceptions and might be limited to some occupational environments (Anwer et al., 2021). On the other hand, biochemical workload markers offer reliable measurements on the individuals' physical exertion (Horta et al., 2019) but do not allow a continuous non-invasive examination during normal working activities.

To overcome these limitations, researchers have attempted to use various physiological variables such as heart rate, skin temperature, electromyography and jerk metrics to monitor real-time fatigue in any occupational setting (Friedl, 2018; Lee et al., 2017). However, this obtained information needs to be translated into actionable data that can actually be used to prevent further physical impairments and improve individuals' performance in the workplace. In this regard, an algorithm for fatigue detection and continuous assessment through physiological monitoring was used, and the preliminary results of its application within military training events are presented in this study.

\section{METHODOLOGY}

\section{Participants}

Two male subjects were randomly selected to participate in the study and, their anthropometric characteristics are detailed in Table 1. They were regular elements from the Portuguese Army undertaking the 131st Commando Course (from April to June 2018). Before investigative procedures, recruited participants gave their written consent and were briefed on the purpose, potential risks, and benefits of the experiences. Additionally, they underwent a medical examination (as a standard requirement to take part in the course) and did not present cardiac, vascular, pulmonary, or any allergic diseases; they were considered mentally healthy and were not prescribed any regular medication.

Table 1. Participants

\begin{tabular}{ccc}
\hline Subject & A & B \\
\hline Age & 21,0 & 24,0 \\
Height $(\mathbf{c m})$ & 178 & 188 \\
Weight $(\mathbf{k g})$ & 76,05 & 86,38 \\
Body fat mass (kg) & 11,48 & 6,78 \\
Body fat (\%) & 15,09 & 7,84 \\
Body Mass Index $\left(\mathbf{k g} / \mathbf{m}^{2}\right)$ & 24,03 & 24,43 \\
\hline
\end{tabular}

\section{Experimental design}

Physiological recordings were continuously collected from each volunteer while participating in the three events. Contrarily to a previous laboratory experience (Bustos et al., 2019), in which participants performed a maximal exertion test, in this case, there were not predefined experimental protocols, and recordings were gathered during normal training conditions. After obtaining the results from the algorithm, details about each event were asked and correlated with the performed assessment. Prior to the training events, participants donned the physiological monitoring system, as indicated. The three evaluated events are briefly described lines below. 
PIC (April 26th, 2018): Acronym of 'Pista Individual de Combate' (Portuguese words for 'Individual combat track'), this test consists of individual combatant progression under real fire. It is composed of 17 periods, in which activities of wounded displacement, a tunnel passage, lifeguarding, combat, and communication during stressful simulated conditions are included.

MARCOR (June 4th - 5th, 2018): Denominated by combining the Portuguese words: 'MarchaCorrida' (March-Run), this physical test involves running and marching with loads within a $42 \mathrm{~km}$ path. Approximate completion duration varies from 4 to 6 hours.

PCC (May 24th, 2018): Similar to the PIC, this test denominated 'Pista Coletiva de Combate' (Portuguese words for 'Collective Combat Track') is composed of various activities divided into 21 periods. However, in this case, individuals are part of different groups, and tasks are oriented to test performance and teamwork. With all, of the three events covered, this one involved the least physical effort for participants.

Since sensors were randomly assigned to participants before each test, it was not possible to obtain data from the same subject during the three tests. As a result, Participant A was monitored during the PIC test, while participant $B$ was assessed while performing both MARCOR and PCC tests.

\section{Equipment}

During the three events, physiological measurements were recorded every 15 seconds through a chest belt physiological monitoring system: EquiVital LifeMonitor equipment (Hidalgo Ltd., Cambridge, U.K.), a "wear and forget" system type already validated for research purposes (Liu et al., 2013). For core temperature recordings, ingestible thermometer pills from Vital Sense were used. These pills travel along the digestive tract harmlessly and leave the body naturally within 24 to 72 hours. Finally, body composition was assessed using bioelectrical impedance analysis with the Body Composition Analyzer InBody230 (Karelis et al., 2013).

\section{Data analysis}

Physiological monitoring records were assessed using an algorithm (implemented using Python 3.6) that enables analysis and provides an integrated assessment of variables per minute. This algorithm has already been described and tested in a previous study (Bustos et al., 2019). Its main feature lies in classifying received sensory information into health alarm levels that refer from a good overall health status (alarm 1) to four different levels of fatigue (alarms 1.5, 2, 3 and 4). The system also attributes a warning when the sensor is not functioning correctly (alarm -1). The default number of examined physiological variables is three (heart rate, breathing rate, and core temperature), another two referring to body position and the last one determining the validity of the sensor recordings. However, if one or more of these variables are found to be 'noisy' (with unreliable values), they are filtered, and the algorithm assesses the rest of the available information. Nevertheless, the more of these variables are included, the higher the reached accuracy.

\section{RESULTS AND DISCUSSION}

The proposed method has been evaluated previously in controlled laboratory conditions, during a short maximal exertion test and proved an overall reliable performance (Bustos et al., 2019). In this study, a retrospective assessment throughout three military training events was performed. In general, outcomes demonstrated that despite the diverse activities covered within each event, the algorithm evidenced the different stages of training and the resulting 
physical demands on subjects throughout the exercises. Physiological monitoring files from two subjects participating in three different military events were assessed. In all, the algorithm proved to be a promising approach for detecting physically demanding periods and evidencing the physiological impairment resulting from the most stressful situations, which suggests it can be extrapolated to various occupational and physically intense training settings.

Results were obtained per minute, and Table 2 presents the time percentage under each alarm category for the three cases. Contrary to the referred laboratory experience (Bustos et al., 2019), higher alert levels and a higher percentage of time in those levels were expected since prolonged intermittent exercises are proved to be more physically demanding than short continuous practices (Edwards et al., 1973; Edwards et al., 1972; Kraning 2nd \& Gonzalez, 1991), which was corroborated within obtained assessments. Specifically, outcomes from the PIC test showed an alarm 2 (moderate physical intensity) for $10 \%$ of the test duration. The participant taking this assessment showed a stable tendency, reporting a very minimum percentage under alarm 3 and approximately $88 \%$ of the time under alarm 1 (no fatigue stage). This steadiness could be justified considering his longer experience (compared with the other soldier) in military training practices, which infers the need for assessing fatigue on an individual basis.

Table 2. Time percentage under each alarm level.

\begin{tabular}{lrrr}
\hline Alarm category & Subject A - PIC & Subject B - MARCOR & Subject B - PCC \\
\hline Alarm 1 & 88.32 & 17.41 & 54.64 \\
Alarm 1.5 & 0.00 & 0.00 & 0.00 \\
Alarm 2 & 10.58 & 45.52 & 44.30 \\
Alarm 3 & 1.09 & 29.35 & 1.06 \\
Alarm 4 & 0.00 & 7.71 & 0.00 \\
\hline
\end{tabular}

Similar to the PIC, the PCC results showed a bigger percentage of alarm 1. However, the level of physical exertion was significantly superior since both alarms 2 and 3 lasted longer than in the previous test. Despite this, no health warning was retrieved, and a maximal exertion was observed only $1 \%$ of the time, suggesting the subject was able to deal with all periods of the event. Assessment of more individuals undertaking this test can help validate the physiological cost during this alarm 4 period. On the other hand, from the MARCOR test, it was noted that the highest time percentages under alarms 2 and 3 were retrieved, and the subjects also reported a more significant amount of time under level 4 . Since this test involves mostly an extended physical performance evaluation simulating a marathon, it was anticipated to obtain higher alarm levels and higher time percentages under fatigue. Correspondingly, assessments also revealed the lowest rates under a no fatigue condition (alarm 1). From all, this event proved to be the most physically demanding for soldiers.

Furthermore, when referring to the physiological responses determining the alarms, figures 1 and 2 help to observe how the alert-based system accurately represented these physiological outcomes. Core temperature and heart rate were considered the most discriminating factors when performing assessments and, correlation coefficients between them corroborate these positive associations. On the other hand, outcomes also revealed that there were periods in which heart rate values reached $180 \mathrm{bpm}$, and the breathing rate was above $60 \mathrm{rpm}$, denoting the high physiological cost associated with these practices. However, the most significant values were observed in core temperature. During the march-run series, there were periods in which they went above $39^{\circ} \mathrm{C}$ and maintained those high numbers over several minutes. This fact attests 
to the severe acute stress under which soldiers are exposed and verifies what is evidenced in literature during military field practices (Lieberman et al., 2005; Lieberman et al., 2016; Ralph et al., 2017).

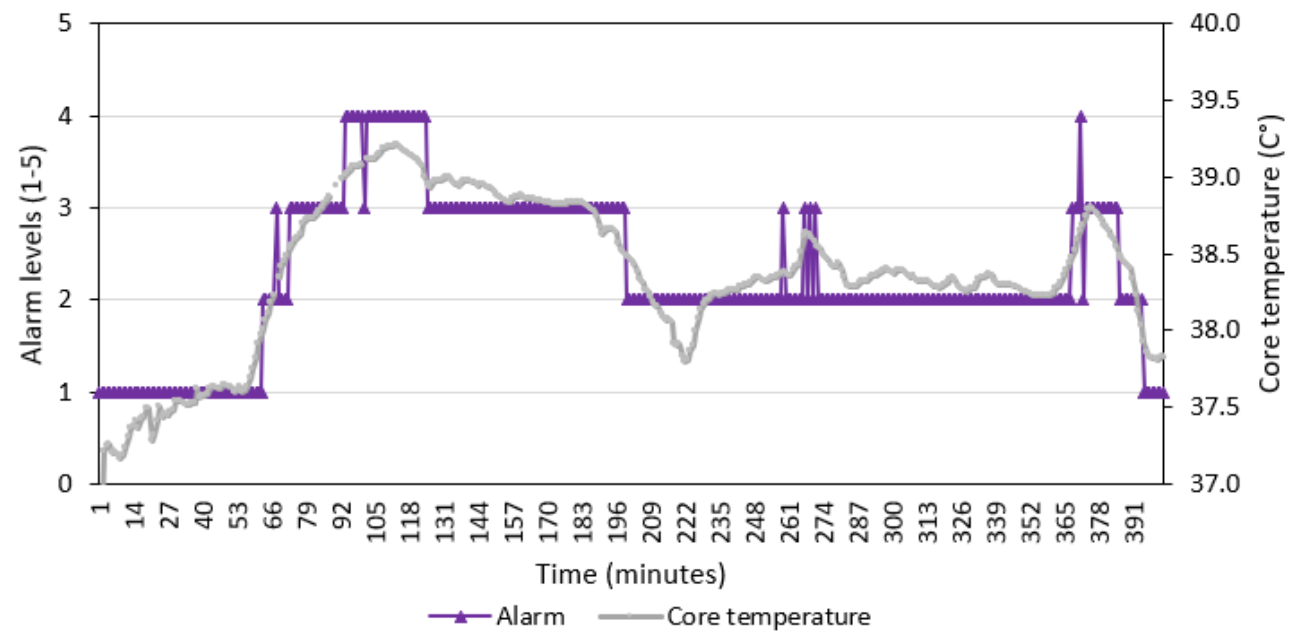

Figure 1. Results vs. core temperature from Subject B during MARCOR test.

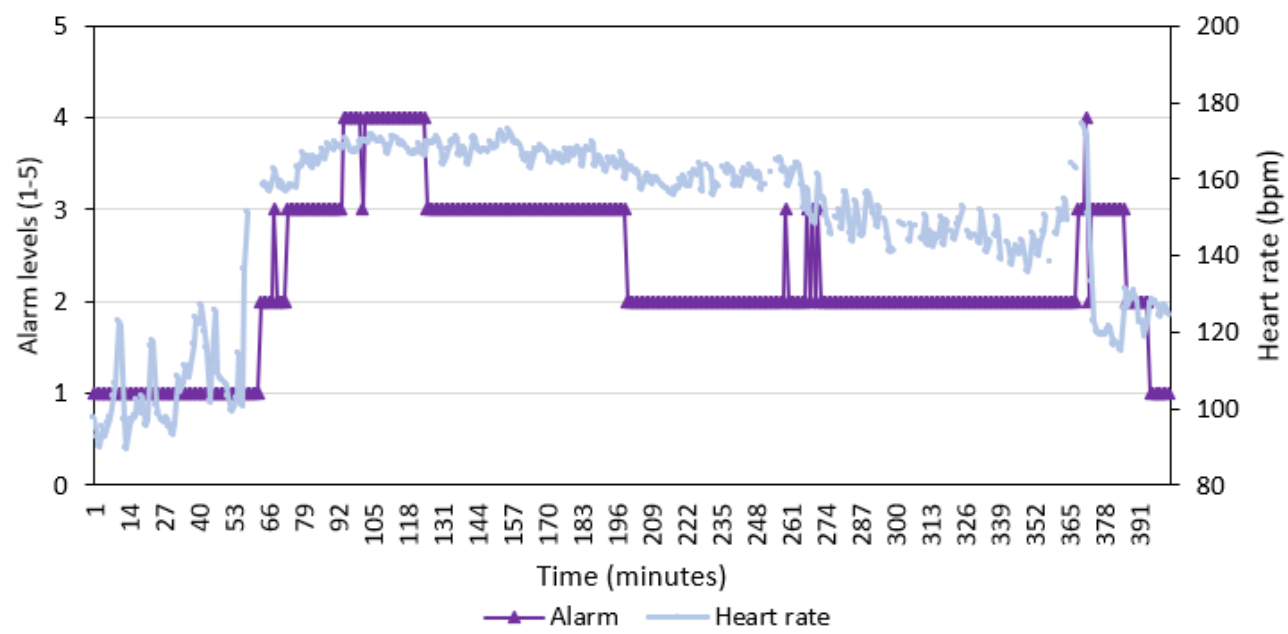

Figure 2. Results vs. heart rate from Subject B during MARCOR test.

\section{Limitations}

Since this study refers to field measurements and was not conducted in controlled laboratory settings, some inevitable limitations were observed. There were differences in testing times and quantity of available information, and some recordings had to be dismissed due to their compromised validity, which did not allow a complete overview of all physiological variables. Furthermore, the small sample referred for assessment did not permit the drawing of generalisable conclusions. Finally, the time course for complete recovery from the training could not be determined since there were no follow-up assessments. However, it was possible to collect data for three specific events each with a unique profile, which allowed to test the algorithm's capacity to detect fatigue in different real-life scenarios.

\section{CONCLUSIONS}

In this study, the preliminary outcomes from the validation of a decision-based algorithm for fatigue detection within training events are reported. Based on these initial results, it was 
demonstrated that the proposed alert-based system was able to provide reliable outcomes and led to the accurate identification of the most physically demanding periods and their direct impact on physiological variables. The assessment of more individuals undertaking these events will help establish the intensity of each training period clearly, leading to a better planning and management of training and work-rest cycles. Overall, by providing a multivariate approach, it is believed that this assessment method, with further modifications, has the potential to improve fatigue management among military populations. For future work, its validity will be tested through a more significant sample, and additional variables will be added to the assessment system for more specificity and robustness.

\section{Acknowledgments}

This work was funded by the Foundation of Science and Technology (FCT Portugal) through the PhD studentship SFRH/BD/143608/2019, the Military Academy Research Center (CINAMIL), and the Associated Laboratory for Energy, Transports and Aeronautics (LAETA). The authors would also like to acknowledge the support of the Doctoral Program in Occupational Health and Safety from the University of Porto.

\section{References}

Anwer, S., Li, H., Antwi-Afari, M. F., Umer, W., \& Wong, A. Y. L. (2021). Evaluation of Physiological Metrics as RealTime Measurement of Physical Fatigue in Construction Workers: State-of-the-Art Review [Review]. Journal of Construction Engineering and Management, 147(5), Article 03121001. https://doi.org/10.1061/(ASCE)CO.1943-7862.0002038

Belenky, G., Lamp, A., Hemp, A., \& Zaslona, J. L. (2014). Fatigue in the Workplace. In Sleep Deprivation and Disease (pp. 243-268). Springer.

Bustos, D., Guedes, J., Alvares, M., Vaz, M., \& Torres Costa, J. (2019). Real Time Fatigue Assessment: Identification and Continuous Tracing of Fatigue Using a Physiological Assessment Algorithm. Occupational and Environmental Safety and Health, In Press. https://doi.org/https://doi.org/10.1007/978-3-030-14730-3_28

Edwards, R., Ekelund, L.-G., Harris, R., Hesser, C., Hultman, E., Melcher, A., \& Wigertz, O. (1973). Cardiorespiratory and metabolic costs of continuous and intermittent exercise in man. The Journal of physiology, 234(2), 481497.

Edwards, R., Melcher, A., Hesser, C., Wigertz, O., \& Ekelund, L. G. (1972). Physiological correlates of perceived exertion in continuous and intermittent exercise with the same average power output. European journal of clinical investigation, 2(2), 108-114.

Fang, D., Jiang, Z., Zhang, M., \& Wang, H. (2015). An experimental method to study the effect of fatigue on construction workers' safety performance. Safety science, 73, 80-91.

Friedl, K. E. (2012). Predicting human limits-the special relationship between physiology research and the Army mission. Friedl KE, Santee WR (Eds) Military Quantitative Physiology: Problems and Concepts in Military Operational Medicine: Problems and Concepts in Military Operational Medicine, 1-38.

Friedl, K. E. (2018). Military applications of soldier physiological monitoring. Journal of Science and Medicine in Sport. Hallowell, M. R. (2010). Worker fatigue. Professional safety, 55(12), 18.

Horta, T. A. G., Bara Filho, M. G., Coimbra, D. R., Miranda, R., \& Werneck, F. Z. (2019). Training Load, Physical Performance, Biochemical Markers, and Psychological Stress During a Short Preparatory Period in Brazilian Elite Male Volleyball Players [Article]. Journal of strength and conditioning research, 33(12), 3392-3399. https://doi.org/10.1519/JSC.0000000000002404

Karelis, A. D., Aubertin-Leheudre, M., Duval, C., \& Chamberland, G. (2013). Validation of a portable bioelectrical impedance analyser for the assessment of body composition [Article]. Applied Physiology, Nutrition and Metabolism, 38(1), 27-32. https://doi.org/10.1139/apnm-2012-0129

Kraning 2nd, K., \& Gonzalez, R. R. (1991). Physiological consequences of intermittent exercise during compensable and uncompensable heat stress. Journal of Applied Physiology, 71(6), 2138-2145.

Lee, K. A., Hicks, G., \& Nino-Murcia, G. (1991). Validity and reliability of a scale to assess fatigue. Psychiatry Research, 36(3), 291-298. https://doi.org/https://doi.org/10.1016/0165-1781(91)90027-M

Lee, W., Lin, K.-Y., Seto, E., \& Migliaccio, G. C. (2017). Wearable sensors for monitoring on-duty and off-duty worker physiological status and activities in construction. Automation in Construction, 83, 341-353.

Lerman, S. E., Eskin, E., Flower, D. J., George, E. C., Gerson, B., Hartenbaum, N., Hursh, S. R., \& Moore-Ede, M. (2012). Fatigue risk management in the workplace. Journal of Occupational and Environmental Medicine, 54(2), 231-258. 
Lieberman, H. R., Bathalon, G. P., Falco, C. M., Kramer, F. M., Morgan, C. A., \& Niro, P. (2005). Severe decrements in cognition function and mood induced by sleep loss, heat, dehydration, and undernutrition during simulated combat. Biological psychiatry, 57(4), 422-429.

Lieberman, H. R., Farina, E. K., Caldwell, J., Williams, K. W., Thompson, L. A., Niro, P. J., Grohmann, K. A., \& McClung, J. P. (2016). Cognitive function, stress hormones, heart rate and nutritional status during simulated captivity in military survival training. Physiology \& behavior, 165, 86-97.

Liu, Y., Zhu, S. H., Wang, G. H., Ye, F., \& Li, P. Z. (2013). Validity and reliability of multiparameter physiological measurements recorded by the Equivital LifeMonitor during activities of various intensities. Journal of occupational and environmental hygiene, 10(2), 78-85.

Parnell, N., Rye, K., \& Greenberg, N. (2018). Health and well-being management in the military: a systematic review of genetic studies. Journal of the Royal Army Medical Corps, 164(4), 302-308. https://doi.org/10.1136/jramc-2017-000765

Rachmawati, S., Aktsari, M., Suryaningsih, A., Hawali Abdul Matin, H., \& Suryadi, I. (2020). Assesment Work Fatigue to Workers in Environment Underground Mining Areas Based on Fatigue Assesment Scale Questionnaires. E3S Web of Conferences,

Ralph, C. S., Vartanian, O., Lieberman, H. R., Morgan, C. A., \& Cheung, B. (2017). The effects of captivity survival training on mood, dissociation, PTSD symptoms, cognitive performance and stress hormones. International Journal of Psychophysiology, 117, 37-47.

Ream, E., \& Richardson, A. (1996). Fatigue: a concept analysis. International journal of nursing studies, 33(5), 519-529.

Stacey, M. J., Hill, N., \& Woods, D. (2018). Physiological monitoring for healthy military personnel. In: British Medical Journal Publishing Group.

Zhang, M., Sparer, E. H., Murphy, L. A., Dennerlein, J. T., Fang, D., Katz, J. N., \& Caban-Martinez, A. J. (2015). Development and validation of a fatigue assessment scale for US construction workers. American journal of industrial medicine, 58(2), 220-228. 